\title{
Simulation Study of Feedback based Adaptive TCP Protocol for improving the Performance of TCP and High Speed Data Transmission Based on Congestion Window Size
}

\author{
Ramesh Pusuluri $^{1 *}$, Reena Aggarwal ${ }^{2}$ and Sivachandar ${ }^{3}$ \\ ${ }^{1}$ Assistant Professor, Department of Electronics \& Communication, Vel Tech \\ University, Tamilnadu, India \\ ${ }^{2}$ Assistant Professor, Department of Electronics \& Communication, Lovely \\ Professional University, Punjab, India \\ ${ }^{3}$ Assistant Professor, Department of Electronics \& Communication, Vel Tech \\ University, Tamilnadu, India \\ rameshpusuluri999@gmail.com, ${ }^{2}$ reena.16120@lpu.co.in, ${ }^{3}$ siva5446@gmail.com
}

\begin{abstract}
The major aim of this research work carried out on Ad hoc networks is to develop a model which improves the performance of Transmission Control Protocol (TCP) compared to the existing TCP. On the basis of the research work presented by different researchers all over the world, a method was implemented and it combines feedback based approach as well as the adaptive approach to enhanceTCP performance. To get TCP with enhanced performance, the working of standard TCP was changed to either Full Congestion window-TCP or Partial Congestion window TCP. From the simulation it was found that Partial Congestion window-TCP is more suitable for lossy links like internet. It is found that Full Congestion window TCP is more suitable and appropriate in high usage of bandwidth applications. This new approach combines the adaptive back-off with feedback scheme to make TCP to work better than traditional TCP. This research brought the intelligence wherein this protocol will be able to detect the conditions of the network as well as the various external factors like power and then choose whether to use Full Congestion window TCP or Partial Congestion TCP.
\end{abstract}

Keywords: MANETs, TCP Performance, Feedback Based, Throughput, Transmission Errors

\section{Introduction}

Now a days, the internet has changed the way the public uses to communicate and it's achievement is mostly because of its consumption and simple nature of TCP/IP which is the main mechanism for communication [1]. The standard of General De-facto for transport layer in network is TCP because of its end to end reliability. The applications like video, voice, etc uses User datagram protocol which does not require reliable end to end delivery. To modify conditions in the network, TCP encompasses mechanisms for congestion control and was developed to respond in robust way.

To give communication at high quality, it is necessary to choose effective routing protocols. The resource constraints in the network device are weight, probability and size [2]. Mobile ad hoc network nodes have restricted bandwidth, space of the buffer and battery power. MANET routing protocols have to equally distribute routing tasks in the unfixed hosts. Unfixed devices are embedded by fixed devices in MANETs for

Received (October 19, 2017), Review Result (December 6, 2017), Accepted (December 12, 2017)

${ }^{*}$ Corresponding Author 
communication. The major characteristics of MANETs contains self-configuring flexibility, coordinating in a non-centralized way, radio communication which is multihop, deploying in a rapid manner, breaking of links very frequently due to resource constraints and dynamic topology of mobile nodes [3]. The major requirement of routing protocol in MANET is that it should be completely adaptive, frequent topology change distribution, low computation and maintenance, using resources optimally, optimal free routes and loops.

The main purpose of this work is to find the solution for improvement in performance to use TCP in MANETs. It mainly focuses on several protocols which will be implemented fully to remove fallbacks and should be able to path regaining in case of fall back [4]. As, there is no exact enhancement of the existing protocols to improve transport protocol in Ad hoc networks, this work is providing some optimal ways one can implement to strengthen TCP in unreliable environments in a robust ways.

\section{Literature Survey}

Anantharaman [1] studied the TCP performance of MANETs and showed a set of outcomes of simulation and found the major issues which impact TCP performance in MANETs. They used multiple parameters for link failure detection, route latency evaluation, packet level route index unavailability and flow level route to find the mobility effect. By evaluating the rate of loss, throughput and time taken for retransmission of data, how the mobility effect different performance metrics of TCP and it was compared with each other. The author has proved from the simulation outcomes that how TCP performance was improved in MANETs. It was observed that the author improved the throughput in stack of default protocol by around $75 \%$.

Gavin Holland [2] presented that how TCP performance is affected due to link breakage. With the help of simulation, the author has proved that the throughput has decreased if the node does not know the reason behind the packet drop whether it is due to failure of the route or congestion in the network. The author has made a comparison for multi-hop networks in terms of throughput. The author concluded that the notification of failure of the link has improves the performance of TCP.

In hybrid MANET, certain aspects regarding the TCP performance was described by Karlsson [3] with extensive simulation. In their work, for connecting to public internet unfixed nodes adopt paths of multi hop. Such vision has very interest in $4 \mathrm{G}$ operations as hotspot coverage will be extended.

To examine the cross layer based techniques of TCP for controlling the congestion in MANET, Shukla [4] has carried out extensive survey in MANETs. The TCP assumes the loss of packet was because of presence of network errors which was unacceptable in the networks of unfixed type. If the communication is not disturbed in the network then the performance of the TCP will be better. In this research work, the major factor which degrades the TCP performance in ad hoc networks was found. In particular there are few factors like mobility, wireless channels, frequent changes of routes; link breakages are taken which degrade TCP performance. It is concluded that if the communication was not of disturbed type, then TCP performs better.

Most of the times, TCP is used for traffic flow in wired networks. In wired networks the loss of the data is always due to the congestion in the network. In Ad hoc networks, loss of data was not always due to congestion in network. Ahmad Al Hanbali [5] presented in his paper about various reasons for the information loss in Ad hoc networks like lossy nature of channels, Hidden and exposed terminals, Asymmetry due to path, Partitioning of the network, Routing Failure and limited available power.

To reduce unnecessary transmissions and loss of throughput in MANETs a feedback scheme was introduced by KartikChandran[6]. When the link was broken, the node of source will be informed with a message of the failure of the route by sending a packet, 
after receiving this message the source node will stop sending the data packets and the timers of the source will be in resumed state for some amount of time. When the route from intermediate nodes to destination node is again established, the intermediate nodes will inform the source node with a notification of route re-establishment packet, after that it continues transmitting the packets by unfreezing timers. Feedback based TCP gives best performance for simulated results.

As the mechanisms of TCP for controlling congestion are introducing the very high flow of traffic on present communication networks which degrade the performance of the network. To overcome this problem, Amit Aggarwal [7]proposed a technique which space the data evenly and send that data into the network in the complete Round trip delay time (RTT), by sending the data in this way will leads to reduction in burst flow of traffic. With Pacing of the data, better throughputs, less packet loss and enhanced performance can be achieved.

In lossy wireless networks, to improve the TCP network performance Thomas [8] has introduced various models for effective throughput and utilization of bandwidth. The author has simulated on multi-stream bandwidth, throughput and packet loss. The TCP connections which are connected in parallel is having effective throughput and utilization of the bandwidth. It is proved by the author that if the congestion is not present, the usage of parallel connections of TCP is equal to that of single connection of Multi stream source.

The network congestion is the main reason for the loss of packets which was not completely acceptable in case of wireless networks stated by Xiang [9]. The major factors which degrade the performance of TCP in wireless communication are bit errors, handoffs in case of single-hop networks, medium access contention, changing the routes frequently and breaking of the routes in multiple-hop networks. The performance of single-hop networks is better than multiple-hop networks. TCP should be able to differentiate congestion and route/link breakages.

For improving the performance of the TCP, the congestion in the network is handled in efficient manner by comparing the two variants of the TCP which are HS-TCP and TCP Reno by Gururaj [10]. The author has performed the simulation in NS2 as well as the mathematical calculation for both HS-TCP and TCP Reno and proved that HSTCP is better.

\section{Problem Definition}

MANETs contains unfixed multi-hop links that faces frequent packet loss due to property of erroneous unfixed channels, route breakages and contention of MAC layer. The performance of the TCP is very poor if it assumes these problems as losses in congestion and invokes congestion control algorithm [5]. Unlike some networks which are fixed, MANETs have some different features which degrade TCP performance. Such features include a non-predictable, unfixed channel which occurs due to hidden terminal issues, collisions due to random access, exposed terminal issues, frequent breakage of routes due to the mobility of the nodes.

As most of these issues create problems to TCP for achieving end-to-end accurate communications in MANETs. So, the major focus on this research work is to develop a solution for TCP in ad hoc networks to improve the performance. Asymmetric nature of MANETs is one of the factor that affects the performance of TCP [6]. In single-hop wireless networks, the wireless link between mobile terminal and base station is asymmetric in nature. The mobile terminal has limited power when compared to base station as well as the mobile terminal has limited buffer space and processing capability. Multi-path routing is one of the main dis-advantages in MANET [7]. MANET routes are short lived because of breaking of links very frequently. 
To decrease delay caused by route re-computation, some of the protocols for routing maintain different routes among a pair of sender and receiver and utilize multi-path routing for transmitting the packets. In those cases, packets which are coming from different paths will not come in order at the receiver [8]. Those arrivals of packets which are out-of-order will be assumed as congestion by TCP receiver as TCP is not ware of multi-path routing. After this, the receiver will generate acknowledgements which are duplicating. These acknowledgements cause the sender to initiate fast retransmission by invoking congestion control algorithms.

\section{Research Design}

The main aim of this research is to develop a solution which improves the performance of the TCP using feedback and adaptive nature which gives better performance than traditional TCP. The proposed research methodology introduces feedback based approach for improving the performance of the TCP. This methodology can be implemented using NS-2 Simulator and various performance parameters like Throughput, Good-put, Round trip delay time (RTT) can be measured. It proposes modifications to the traditional TCP for improving performance in ad hoc wireless networks. TCP feedback requires the support of a reliable link layer and a routing protocol that can provide feedback to the TCP sender about the path breaks. The routing protocol is expected to repair the broken path within a reasonable time period.

TCP feedback aims to minimize the throughput degradation resulting from the frequent path breaks that occur in ad hoc wireless networks. During a TCP session, there could be several path breaks resulting in considerable packet loss and path reestablishment delay. Upon detection of packet loss, the sender in a TCP session invokes the congestion control algorithm leading to the exponential back-off of retransmission timers and a decrease in congestion window size. The implemented feedback based TCP protocol is classified into two types used for different applications which are Full Congestion window TCP termed as Fcwnd-TCP and Partial Congestion window TCP termed as Pcwnd-TCP which are briefly described as below.

\subsection{Full Congestion Window TCP}

The advantage of Full Congestion window TCP (Fcwnd-TCP) is the utilization of the high bandwidth of the network. When compared to standard TCP, Full congestion window TCP (Fcwnd-TCP) have the support of big-sized congestion window. Full congestion window TCP (Fcwnd-TCP) modifies the size of the window which was released and utilized due to coming of the congestion which is motive of the total window size. If the window size is small, then Full Congestion window TCP (Fcwnd-TCP) will works as similar to basic TCP. If window size is large then Full Congestion window TCP (Fcwnd-TCP) increment window size by choosing value based on exact window size in the functionality.

These changes eliminate the slow working of ordinary TCP. Full Congestion window TCP (Fcwnd-TCP) works well and it permits the full consumption of multiple gigabit links and high delay. On the basis of the magnitude of window size, the increase in larger size and decrease in smaller size of the window makes the Full Congestion window TCP (Fcwnd-TCP) to give better performance than the ordinary TCP. This approach makes high-speed ad hoc links to full utilization and it does not degrade the advantages of the ordinary TCP. The basic feedback based TCP model with nodes P, Q, R, S is as shown in the figure below. Even when Full Congestion window TCP (Fcwnd-TCP) share bandwidth with the ordinary TCP connections, the performance is still good. Multiple connections of the ordinary TCP were having almost the same performance of the single connection of the Full Congestion window TCP in terms of controlling the congestion. Some protocols which are non-TCP can delay the connections of the ordinary TCP which 
are not TCP-friendly. The following Figure 1 shows implementation of feedback based TCP with four nodes $\mathrm{P}, \mathrm{Q}, \mathrm{R}$ and $\mathrm{S}$.

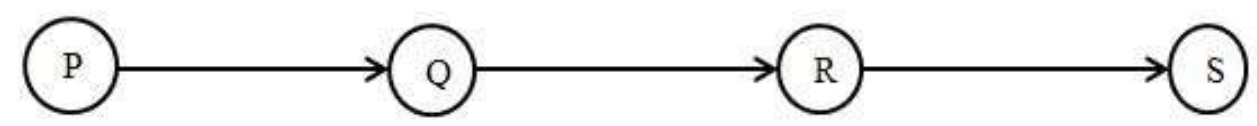

(a) TCP-F connection from $P$ to $S$

TCP state-Snooze

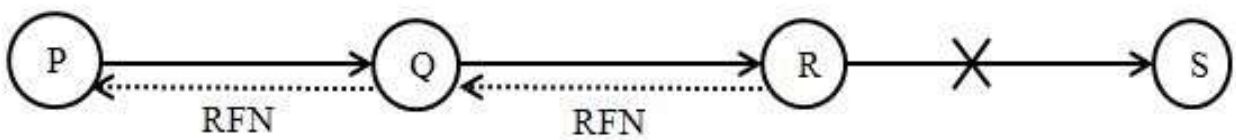

(b) Link $\mathrm{R}$ to $\mathrm{S}$ breaks and $\mathrm{R}$ originates Route failure notification

TCP state-Connected

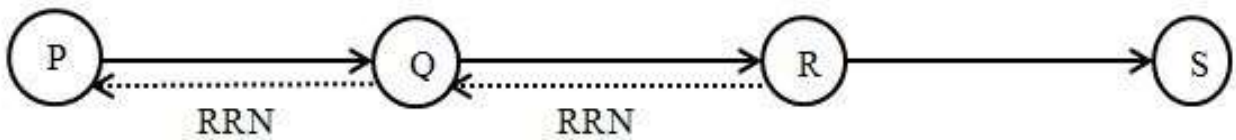

(c) Link R-S rejoins and R originates Route Re-establishm ent Notification

Figure 1. Implementation of Feedback based TCP with Nodes P, Q, R, S

When the source node receives the acknowledgement from the destination node then the source node will evaluate the Round trip delay time (RTT) and this value is stored in the RTT Table. Using RTT table, the average and maximum values are found and it is used to calculate the bandwidth availability. From the minimum RTT value and the RTT value, arrival and the expected rate of the connection is calculated. From the RTT differences, the variation will be estimated and if the arrival rate is high or the variation is high, then the size of the window will be adjusted. With the consecutive value of the window, the state of the window will be identified whether it is open, variant, close. If the window is in the state of open, then average variation of the window will be computed from the last point at which it is opened. If window is in the state of invariant, then the average variation of the window will be computed from the last open point to the point it is in the closing state. Value of the change will be calculated. If it is little change, then normal functioning of TCP will be used to enhance forwarding of data. If variation is more, then the size of the window was adjusted to highest size and the execution of window is done.

\subsection{Partial Congestion Window TCP}

Partial Congestion window TCP (Pcwnd-TCP) allows nodes to achieve maximum throughput having critical situations of high congestion. This kind of TCP decreases link breaks. In case of normal TCP, it typically results in underutilization. This protocol removes the congestion control algorithm of TCP. Therefore it permits the connection to more saturation by reducing the chances of connection underutilization. Partial Congestion window TCP (Pcwnd-TCP) protocol is suited for the situations which encompass the connections having more packet drop. Partial Congestion window TCP 
(Pcwnd-TCP) behaves like normal TCP which does not have congestion control challenges.

Partial Congestion window TCP (Pcwnd-TCP) supports the traffic of the network as fast as it can. Once the data of the TCP is sent then the acknowledgement of the packet reached from receiver node. When sender got reply it calculates delay, upon storing those values in the table. Using the table, the maximum, average values can be found. Multiplication of those values with the bandwidth of connection evaluates the availability of bandwidth. With these values bandwidth was calculated. From the present size of the window and the value of the bandwidth, the frame setting value will be computed. If present size of window size was larger than medium sized window and unutilized value of band-width, then the frame was adjusted to dynamic window size. If the present window size was lesser than medium window size and medium unused bandwidth, then it is adjusted to desired hint window else, it was adjusted to another mode of operation. As per value of class, the TCP was checked of either under-usage or full usage.

\section{Simulation Model}

For analyzing the basic TCP behavior with mechanism of the proposed adaptive feedback based TCP, a simulation model was designed in network simulator (NS2) which includes sending node, receiving node, routers acts as intermediate nodes and congestors. Congestor will induce the packets of dummy type into the routers with a specific rate of arrival, by this way the traffic load can be controlled in the network. The link controller controls these routers separately and the connection between these routers was established with the help of links which are bidirectional in nature.

The link controller will periodically break the links periodically by keeping the link broken, again connected after some amount of time. Route will be randomly selected and the chances of the failure of each link are equal. While routing the packet from the nodes or hops, if a router observes a link failure, then the packet will be dropped at that instant. If feedback is using at this instant, then the router will forward the messages as notifications to the sender. High amount of priority will be given to these kinds of messages; so, they will not be maintained in queue with normal packets. The simulation model is as shown in Figure 2. This model will analyze the effect of change in network topology.

The router's load will be controlled by changing the rate of the generation of the packets. The delay for reestablishing the routes will show the network capability how it is responding for the changes in the topology. By changing the delay of re-establishment of routes and rate of arrival of congestion, the performance of transport protocols was compared with the network layer function performance by changing the values of network traffic load. In this simulation model, packet size of 100 bytes and rate of data transmission of $10 \mathrm{~kb} / \mathrm{s}$ were used. Between the source and destination, around 12 hops are created and the size of the window is adjusted to 10 kilo bytes which accommodate approximately 50 packets. The protocol used for routing was AODV as it was the most efficient and best routing among all protocols for routing in the Mobile Ad hoc network. TCP agents are attached to different nodes. Sink nodes as well as the source nodes are defined with their positions in flat grid topography of xy plane in Network simulator.

The theoretical procedure for calculating the size of the congestion window is follows.

1) The congestion window size of the TCP will be increased after the reception of the packet of acknowledgement. In this case, the size of the congestion window is:

$\mathrm{CWS}=\mathrm{CWS}+\mathrm{A}(\mathrm{CWS}) /(\mathrm{CWS})$

2) The congestion window size of the TCP will be decreased when the packet was loss in the process of transmission. In this case, the size of the congestion window is:

$\mathrm{CWS}=(1-\mathrm{B}(\mathrm{CWS})) * \mathrm{CWS}$

Where $\mathrm{A}(\mathrm{CWS})$ is the increased congestion window size during Round trip delay time 
$\mathrm{B}(\mathrm{CWS})$ is the decreased congestion window size during Round trip delay time CWS stand for "Congestion Window Size"

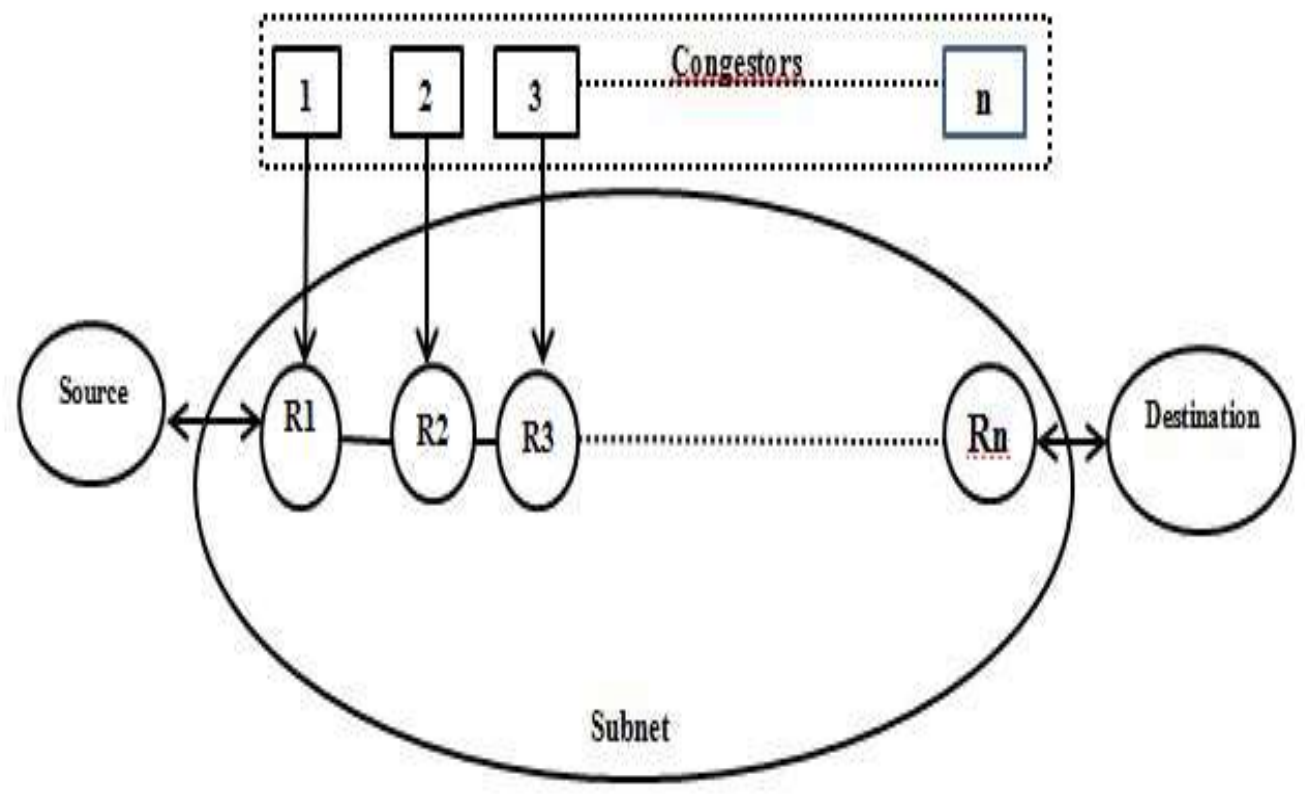

Figure 2. Simulation Model of Feedback based TCP Protocol with Nodes and Routers

$\mathrm{A}(\mathrm{CWS})=2 \mathrm{CWS}^{2} \cdot \mathrm{B}(\mathrm{CWS}) \cdot \mathrm{p}(\mathrm{CWS})$

$(2-b(C W S))$

$\mathrm{B}(\mathrm{CWS})=\frac{\left\{\log (\mathrm{CWS})-\log \left(\mathrm{Wl}_{\text {ow }}\right)\right\}\left(\mathrm{b}_{\text {high }}-0.5\right)}{\log \left(\mathrm{W}_{\text {high }}\right)-\log \left(\mathrm{W}_{\text {low }}\right)+0.5}$

Where $\mathrm{p}(\mathrm{CWS})$ is the rate of packet drop for congestion window $\mathrm{W}_{\text {low }}$ is the high speed data lower bound

$\mathrm{W}_{\text {high }}$ is the high speed data upper bound

$b_{\text {high }}$ is the decrease in the size at the largest congestion window

\section{Results and Discussion}

The Full Congestion Window-TCP (Fcwnd-TCP) and Partial Congestion Window TCP (Pcwnd-TCP) are the sub-classification of Feedback implemented with the adaptive backoff response and the classification is done on the effective utilization of the available window size of congestion. The following Figure 3 in the form of graph shows the Throughput which is taken on y-axis versus number of nodes which is taken on $\mathrm{x}$-axis for Full size congestion window TCP and Partial size congestion window TCP. Here, it was shown that Throughput is directly proportional to number of nodes. With the increase in the number of nodes, the Throughput increases for both Full size congestion window TCP and Partial size congestion window TCP. And the Throughput decreases as number of nodes decreases for both the Full size congestion window TCP and Partial size congestion window TCP. For the same number of nodes, the Throughput is more for Fcwnd-TCP when compared to Pcwnd-TCP. 


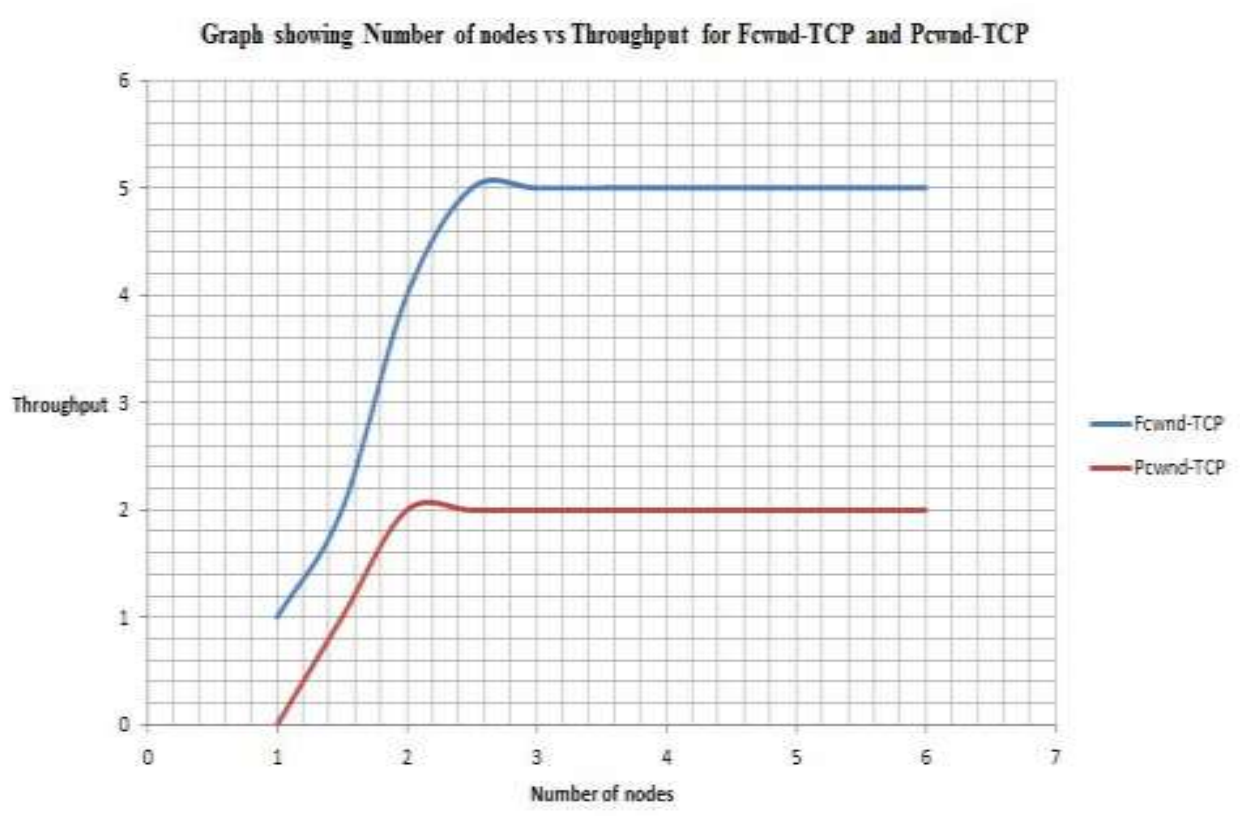

Figure 3. Graph for Throughput vs Number of Nodes for Fcwnd-TCP and Pcwnd-TCP

The following Figure 4 in the form of graph shows the Delay which is taken on y-axis versus number of nodes which is taken on $\mathrm{X}$-axis for Full size congestion window TCP and Partial size congestion window TCP. Here, it was shown Delay is proportional to number of nodes. As nodes increases, the Delay increases for both Full size congestion window TCP and Partial size congestion window TCP. And the Delay decreases as number of nodes decreases for both Full size congestion window TCP and Partial size congestion window TCP. For the same number of nodes, the Throughput is more for Fcwnd-TCP when compared to Pcwnd-TCP.

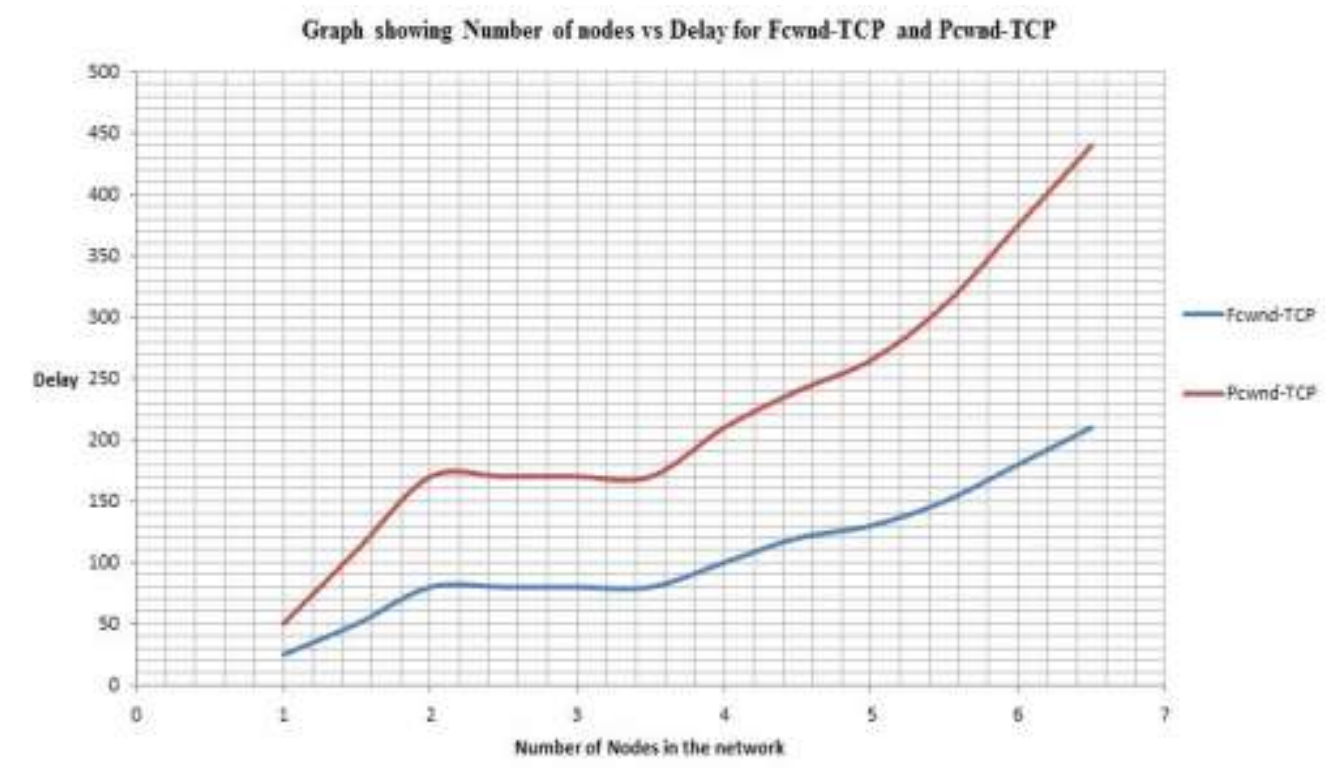

Figure 4. Graph for Delay vs Number of Nodes for Fcwnd-TCP and PcwndTCP 
The following Figure 5 shows the loss of Packet which was taken on y-axis versus number of nodes which is taken on $\mathrm{x}$-axis for Full size congestion window TCP and Partial size congestion window TCP. Here, it was shown that Packet loss was proportional to number of nodes.

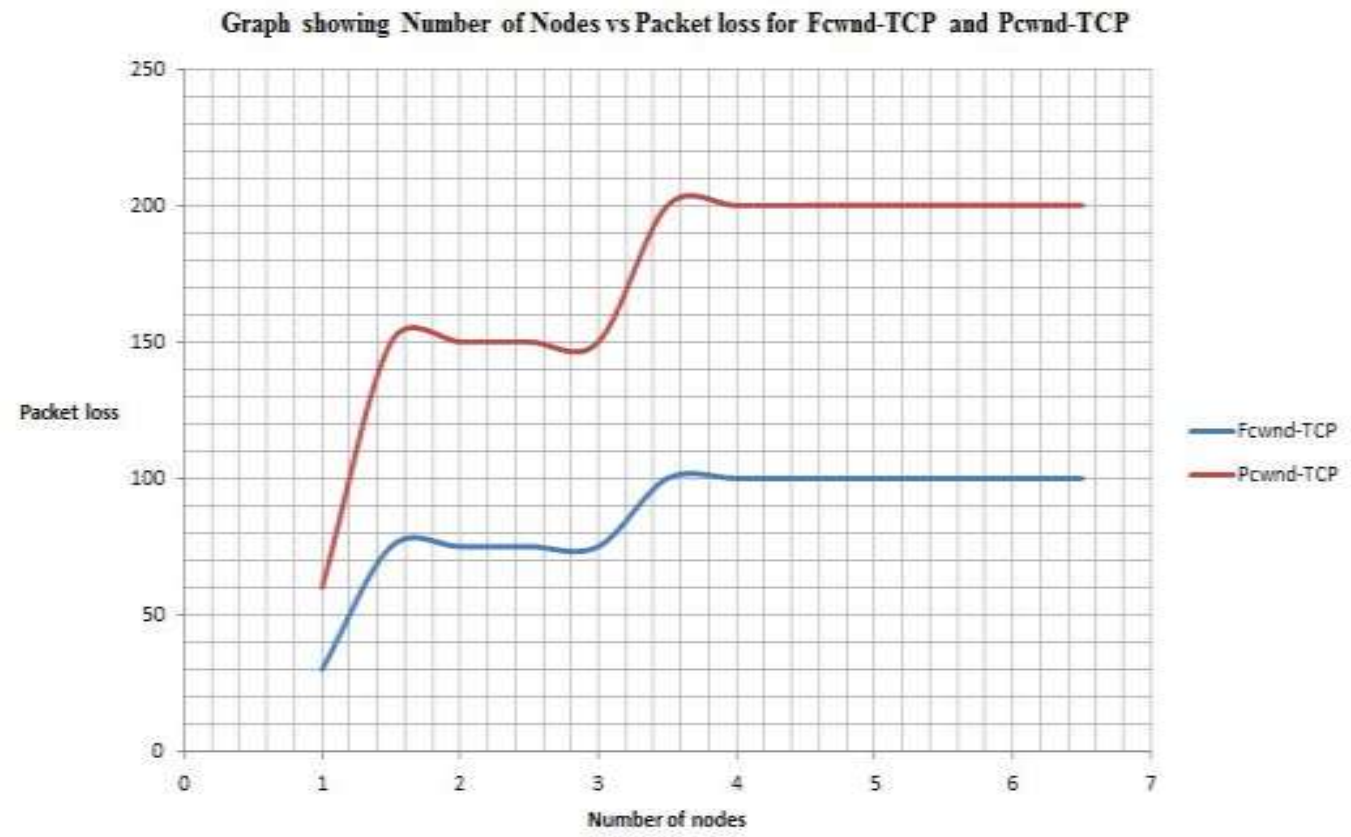

Figure 5. Graph for Packet loss vs Number of Nodes for Fcwnd-TCP and Pcwnd-TCP

As nodes increases, the Packet loss also increases for both Full size congestion window TCP and Partial size congestion window TCP. And the Packet loss decreases as the number of nodes for both the Full size congestion window TCP and Partial size congestion window TCP. For the same number of nodes, the Packet loss is more for Fcwnd-TCP when compared to Pcwnd-TCP. Table 1 shows the comparison of PcwndTCP and Fcwnd-TCP in terms of various performance metrics.

Table 1. Table showing the Comparison of Standard TCP and Feedback based Adaptive TCP

\begin{tabular}{|l|l|l|}
\hline Performance Metric & Pcwnd-TCP & Fcwnd-TCP \\
\hline 1.Throughput & Low & $\begin{array}{l}\text { High compared to Pcwnd- } \\
\text { TCP }\end{array}$ \\
\hline $\begin{array}{l}\text { 2.Bandwidth } \\
\text { utilization }\end{array}$ & $\begin{array}{l}\text { High compared to Fcwnd- } \\
\text { TCP }\end{array}$ & Low \\
\hline 3.Delay & High & $\begin{array}{l}\text { Low compared to Pcwnd- } \\
\text { TCP }\end{array}$ \\
\hline 4.Good put & Very Low & High \\
\hline 5.Energy efficiency & Low & high \\
\hline 6.RTT & High & $\begin{array}{l}\text { Low compared to Pcwnd- } \\
\text { TCP }\end{array}$ \\
\hline
\end{tabular}




\section{Conclusion and Future Scope}

The primary intention of this work was to make the variant of the TCP which gives the enhanced improvement in performance in ad-hoc networks. The altered mechanism of controlling the congestion algorithm for Feedback based TCP has improved performance over the standard TCP in Mobile Ad hoc Networks (MANETs). The simulation is performed using Network Simulator (NS2). This research has utilized the feedback based nature of the TCP as well as the adaptive back-off response to classify the standard TCP as Full Size Congestion Window-TCP and Partial size congestion window TCP using the network parameters like delay, Throughput and bandwidth. It has been observed from the simulation that Partial size congestion window TCP is more convenient for networks having high latency as well as links containing lossy nature like highway driving and utilizing the public Internet. The Full Size Congestion Window-TCP is having high bandwidth efficiency and used for networks having lower values of latency like 4G mobile networks. It is clear from the observed results that the concept of combining Pcwnd-TCP or Fcwnd-TCP together with a combination of adaptive and feedback approach gave superior results on all three parameters of Delay, Throughput and Delivery Packet Ratio when compared to the existing traditional approaches. The current testing had a limitation that the authors manually defined scenarios including traffic patterns. This resulted in optimal conditions for the protocols to demonstrate their behavior.

\section{Acknowledgement}

I express my sincere regards to Reena Aggarwal for her valued guidance. This work was enabled and sustained by her vision and ideas. Her scholarly guidance and invaluable suggestions motivated me to complete this paper work successfully.

\section{References}

[1] V. Anantharaman, S.-J. Park, K. Sundaresan and R. Sivakumar, "TCP performance over mobile ad hoc networks: a quantitative study: Research Articles", Wirel. Commun. Mob. Comput., vol. 4, no. 2, (2004), pp. 203-222.

[2] G. Holland and N. Vaidya, "Analysis of TCP Performance over Mobile Ad Hoc Networks", Wirel. Networks, vol. 8, (2002), pp. 275-288.

[3] J. Karlsson, A. Batlle, A. J. Kassler and B.-S. Lee, "TCP Performance in Mobile Ad Hoc Networks Connected to the Internet", Rev. Cient. Period, vol. 10, no. 1, (2007), pp. 1-3.

[4] N. Shukla, N. Gupta, N. Parveen and M. Scholor, "Survey Of Cross Layer Based TCP Congestion Control Techniques in MANET", Int. J. Emerg. Technol. Adv. Eng. Website www.ijetae.com ISO Certif. J., vol. 4, no. 3, (2014), pp. 446-452.

[5] H. Ahmad, E. Altman and P. Nain, "A Survey of TCP over Ad hoc networks", INRIA B.P. 93, 06902 Sophia Antipolis Cedex, France June, (2005).

[6] K. Chandran, S. Raghunathan, S. Venkatesanand and R. Prakash, "A Feedback Based Scheme For Improving TCP Performance In Ad-Hoc Wireless Networks", Computer Science Program University of Texas at Dallas Richardson, TX 75083-0688.

[7] A. Aggarwal, S. Savage and T. Anderson, "Understanding the Performance of TCP Pacing", 14th Annual IEEE Workshop on Wireless Local Networks 2013 978-1-4799-0540-9/13/\$31.00 @2013 IEEE.

[8] Thomas, Brian and Noble, "The End-to-End Performance Effects of Parallel TCP Sockets on a Lossy Wide-Area Network", International Workshop on Wireless Ad-hoc Network, (2004).

[9] X. Chen, H. Zhai, J. Wang and Y. Fang, "A Survey on Improving TCP Performance over Wireless Networks", 4th International Conferences on Mobile and Wireless Communication Network, (2002).

[10] H. L. Gururaj and Ramesh, "Congestion Control for Optimizing Data Transfer rate in Mobile Ad-hoc Networks using HSTCP", International Conference on Emerging Research in Electronics, Computer Science and Technology, (2015).

[11] Z. Zhenghua Fu, X. XiaoqiaoMeng and S. Songwu Lu, "How bad TCP can perform in mobile ad hoc networks", in Proceedings ISCC 2002 Seventh International Symposium on Computers and Communications, (2002), pp. 298-303.

[12] H. Balakrishnan, V. N. Padmanabhan, S. Seshan and R. H. Katz, "A comparison of mechanisms for improving TCP performance over wireless links”, IEEE/ACM Trans. Netw., vol. 5, no. 6, (1997), pp. 756-769. 
[13] M. Gerla, K. Tang and R. Bagrodia, “TCP performance in wireless multi-hop networks”, in Proceedings WMCSA'99. Second IEEE Workshop on Mobile Computing Systems and Applications, (1999), pp. 4150 .

[14] S. Xu and T. Saadawi, "Does the IEEE 802.11 MAC protocol work well in multihop wireless ad hoc networks?", IEEE Commun. Mag., vol. 39, no. 6, (2001), pp. 130-137.

[15] J. Guo, H. Liu, J. Dong and X. Yang, "HEAD: A Hybrid Mechanism to Enforce Node Cooperation in Mobile Ad Hoc Networks", TSINGHUA SCIENCE AND TECHNOLOGY ISSN 1007-0214 36/49, vol. 12, no. S1, (2007), pp. 202-207

[16] A. Deshpande and A. Kaushal, "MX-TCP and HS-TCP as Possible Options to Overcome TCP Limitations in Multi-Hop Ad-Hoc Networks", International Journal of Advanced Research in Computer and Communication Engineering, vol. 4, issue 12, (2015).

[17] F. Hirose, M. Fukuhara, T. Hatano, H. Shigeno and K.-I. Okada, "A Two-Level ECN Marking for Fair Bandwidth Allocation between HSTCP and TCP Reno", Proceedings of the 25th IEEE International Conference on Distributed Computing Systems Workshops.

[18] A. Deshpande and A. Kaushal, "Feedback-based Adaptive Speedy Transmission (FAST) Control Protocol to Improve the Performance of TCP over Ad-Hoc Networks", 2016 International Conference on Advances in Computing, Communications and Informatics (ICACCI), Jaipur, India, (2016).

\section{Author}

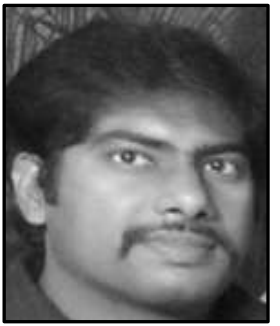

Ramesh, she received his Bachelor of Technology in Electronics from Koneru Lakshmaiah University in 2014 and Master of Technology in Wireless Communication from Lovely Professional University in 2017 in India. At present he is working as assistant professor in Vel Tech University in Chennai. His area of research is Ad hoc networking. 
International Journal of Grid and Distributed Computing

Vol. 11, No. 1 (2018) 